\title{
Dissipative Process as a Mechanism of Differentiating Internal Structures between Dwarf and Normal Elliptical Galaxies in a CDM Universe
}

\author{
Masao Mori \\ Max-Planck-Institut für Astronomie, Königstuhl 17, D-69117 Heidelberg, Germany \\ Institute of Astronomy, University of Tokyo, Mitaka, Tokyo 181-8588, Japan \\ Yuzuru Yoshii \\ Institute of Astronomy, University of Tokyo, Mitaka, Tokyo 181-8588, Japan \\ Research Center for the Early Universe, University of Tokyo, Bunkyo-ku, Tokyo 113-0033, Japan \\ and \\ Ken'ichi Nomoto \\ Department of Astronomy, University of Tokyo, Bunkyo-ku, Tokyo 113-0033, Japan \\ Research Center for the Early Universe, University of Tokyo, Bunkyo-ku, Tokyo 113-0033, Japan
}

\begin{abstract}
We simulate the dynamical, chemical, and spectro-photometric evolution of dwarf and normal elliptical galaxies embedded in a dark matter halo, using a three-dimensional $N$-body/SPH simulation code. For the forming dwarf elliptical galaxies, supernova-driven winds propagating outwards from inside the system collide with the infalling gas and produce the super-shell in which stars are formed. The resulting stellar system forms a loosely bound virialized system due to the significant mass loss and has a large velocity dispersion and a large core. Consequently the surface brightness distribution shows an exponential profile and the color distribution shows a positive gradient such that the colors become redder away from the galaxy center in agreement with observations. On the other hand, for the normal elliptical galaxies with deep gravitational potential, the mass loss out of the system does not have a significant dynamical effect on the stellar system. The resulting surface brightness distribution has a large central concentration like de Vaucouleurs' $r^{1 / 4}$-profile and the color distribution shows a negative gradient as observed. Our simulation shows that different features between dwarf and normal elliptical galaxies stem from different cooling efficiencies for their respective protogalaxies in a standard CDM universe.
\end{abstract}

Subject headings: cosmology: dark matter-galaxies: formation - galaxies: structure - galaxies: abundances

\section{INTRODUCTION}

Recent progress in observational devices and techniques has enhanced our knowledge of formation and evolution of galaxies on a firm statistical basis. The luminosity function of galaxies observed in general fields and in nearby clusters shows a steep slope in both the bright and faint ends, whereas its slope in between exhibits a plateau or a slight dip from $M_{\mathrm{B}}=-19$ to $-13 \mathrm{mag}$ (Loveday 1997; Trentham 1998).

Hierarchical clustering models of galaxy formation in a standard cold dark matter (CDM) universe predict that the number of galaxies monotonically increases with decreasing mass, yielding a power-law 
mass function in a low-mass end (Davis et al. 1985; White \& Frenk 1991; Cole et al. 1994). This implies that, unless the mass-to-light ratio is very different from what we usually expect, an excessive number of dwarf galaxies are predicted beyond that observed in the luminosity function.

In order to remove this serious discrepancy several mechanisms for suppressing the formation of dwarf galaxies have been proposed so far. Efstathiou (1992) proposed an effect of photoionization by ultraviolet background radiation that keeps the gas hot and unable to collapse (see also Chiba \& Nath 1994; Thoul \& Weinberg 1995). Dekel \& Silk (1986) proposed an energy feedback that induces a significant mass loss from low-mass galaxies having the shallow gravitational potential (see also Saito 1979; Yoshii \& Arimoto 1987; Lacey \& Silk 1991).

A clear distinction is seen in structural and photometric quantities between dwarf and normal ellipticals in spite of their morphological similarity. Faber \& Lin (1983) appreciated that dwarf ellipticals have an exponential surface brightness profile rather than de Vaucouleurs' $r^{1 / 4}$-profile applied to normal ellipticals (de Vaucouleurs 1948; Kormendy 1977). Bright dwarfs with $M_{\mathrm{B}}<-16$ mag show a distinct luminosity spike in their center commonly referred to as the nucleated dwarfs (Caldwell \& Bothun 1987; Binggeli \& Cameron 1991; Ichikawa, Wakamatsu \& Okamura 1986), and faint dwarfs do not usually show such a nucleus (Sandage et al. 1985). Young \& Currie (1994) and Binggeli \& Jerjen (1998) showed that the surface brightness profile of dwarf ellipticals changes from an exponential to an $r^{1 / 4}$-like form as their luminosity increases.

Vader et al. (1988) analysed the data from Vigroux et al. (1988) and found that many dwarf ellipticals show systematically redder colors at larger radii away from the galaxy center (Vader et al. 1988; Kormendy \& Djorgovski 1989; Chaboyer 1994; Durrell et al. 1996), which is opposite to the trend observed in normal ellipticals. Vader et al. (1988) interpreted this inverse color gradient in dwarf galaxies in term of the positive gradient of stellar age across the system. However, because of their very low metallicities, stars in dwarf galaxies must have been formed on very short time scales and therefore no appreciable age difference results.

Recent high-resolution observations of nearby dwarf galaxies show a web of filaments, loops and expanding super-giant shells which are imprinted in the gas around the individual galaxies and such a striking feature likely stems from the interaction of interstellar medium with energetic stellar winds from massive stars and/or supernova explosions (Meurer, Freeman \& Dopita 1992; Puche \& Westpfahl 1994; Westpfahl \& Puche 1994; Marlowe, Heckman \& Wyse 1995; Hunter 1996). All these observations motivate us to consider that the energy feedback from supernovae may be of prime importance to understand the discrepancy between the mass function of galaxies predicted from hierarchical models and the luminosity function obtained from recent redshift surveys.

From theoretical viewpoints, while collisionless $N$-body simulations are successful in producing an $r^{1 / 4}$-profile through the merging of galaxies (White 1979) as well as their monolithic collapse (van Albada 1982), only a few simulations incorporating the star formation and energy feedback from supernovae have been performed (e.g. Theis, Burkert \& Hensler 1992; Mori et al. 1997) and in most cases a simple one-zone chemical evolution model has yet been used to interpret the data (e.g. Dekel \& Silk 1986; Yoshii \& Arimoto 1987; Babul \& Ferguson 1996). Navarro, Eke \& Frenk (1996) recently studied the dynamical response of a virialized system to an impulsive mass loss from the galaxy center based on collisionless $N$-body simulations. However, their assumption of impulsive mass loss needs to be justified because it is not known a priori whether the time scale of mass loss is shorter than the dynamical time of the system. Therefore, more realistic simulations are necessary in order to model the evolution of galaxies and properly interpret 
the accumulated data including the spatial gradients of structural and photometric quantities.

In this paper, using a powerful hybrid code for three-dimensional $N$-body and hydrodynamical simulations, we present a unified view of the dynamical, chemical and spectro-photometric evolution of dwarf and normal elliptical galaxies from cosmologically motivated initial conditions. In $\S 2$ we briefly describe the basic equations for the model and the method of incorporating the cooling, star formation, and energy feedback process in the model. More detailed description of the numerical method is deferred to the paper by Mori, Nakasato \& Nomoto (1998). In $\S 3$ we carry out the simulations for dwarf and normal elliptical galaxies originated from a $1 \sigma$ density perturbation in a CDM universe. In $\S 4$ we summarise the result of this paper.

\section{MODELS}

Our three-dimensional simulation method combines the collisionless dynamics with the hydrodynamics in a consistent manner. The evolution of a collisionless system consisting of dark matter and stars is followed by an $\mathrm{N}$-body code, and the hydrodynamical properties of a gaseous component are calculated using the Smoothed Particle Hydrodynamics (SPH) (Lucy 1977; Gingold \& Monaghan 1977). The SPH equations have a similar structure to the self-gravitational $N$-body system. The SPH has no restriction on spatial resolution or deviation from any symmetry and provides a surprisingly accurate result in some applications even with a small number of particles. These characteristics make the SPH most suited to the study of formation and evolution of galaxies.

\subsection{Basic equations}

The gaseous component is described by the fluid equation for a perfect gas in the Lagrangian form. The smoothed average for a hydrodynamical quantity $f(\boldsymbol{r})$ is given by

$$
<f(\boldsymbol{r})>=\int f\left(\boldsymbol{r}^{\prime}\right) W\left(\boldsymbol{r}-\boldsymbol{r}^{\prime}, h\right) d^{3} \boldsymbol{r}^{\prime}
$$

provided $\int W\left(\boldsymbol{r}-\boldsymbol{r}^{\prime}, h\right) d^{3} \boldsymbol{r}^{\prime}=1$ and $\lim _{h \rightarrow 0} W\left(\boldsymbol{r}-\boldsymbol{r}^{\prime}, h\right)=\delta\left(\boldsymbol{r}-\boldsymbol{r}^{\prime}\right)$, where $W(\boldsymbol{r}, h)$ is the smoothing kernel and $h$ is the smoothing length. For nearby gas particles, we replace the integration by summation because of the finite number of fluid elements. For example, the gas density $\rho_{\mathrm{g}}$ at position $\boldsymbol{r}$ is given by

$$
\rho_{\mathrm{g}}(\boldsymbol{r})=\sum_{j} m_{j} W\left(\left|\boldsymbol{r}-\boldsymbol{r}_{j}\right|, h\right),
$$

where $m_{j}$ is the mass of gas particle located at position $\boldsymbol{r}_{j}$. In this paper, following Monaghan \& Lattanzio (1985), we use the standard kernel of spherically symmetric spline.

The momentum equation is given by

$$
\frac{d \boldsymbol{v}_{\mathrm{g}}}{d t}=-\frac{1}{\rho_{\mathrm{g}}} \nabla P+\boldsymbol{g}
$$

and the thermal energy equation associated with the rates of cooling $\Lambda$ and heating $\Gamma$ is given by

$$
\frac{d \varepsilon}{d t}=-\frac{P}{\rho_{\mathrm{g}}} \nabla \cdot \boldsymbol{v}_{\mathrm{g}}+\frac{\Gamma-\Lambda}{\rho_{\mathrm{g}}}
$$


with

$$
P=(\gamma-1) \rho_{\mathrm{g}} \varepsilon,
$$

where $\boldsymbol{v}_{\mathrm{g}}$ is the gas velocity, $\boldsymbol{g}$ is the gravitational force, $P$ is the gas pressure, $\gamma(=5 / 3)$ is the adiabatic index, and $\varepsilon$ is the specific internal energy. We assume that the gas is optically thin and in ionization equilibrium. In order to focus mostly on the heating by energy feedback from supernovae to the interstellar medium, we neglect the effect of photoionization by UV background radiation for simplicity. We use the radiative cooling taken from Table 6 of Sutherland \& Dopita (1993). In Fig. 1 the cooling rate is plotted against the temperature for different metallicities. The top curve shows the cooling rate for $[\mathrm{Fe} / \mathrm{H}]=+0.5$, and the lower curves show those with decreasing the metallicity at intervals of 0.5 dex. The bottom curve corresponds to the zero metallicity for the primordial gas composition.

The equation of motion for a collisionless particle of either dark matter or star at $\boldsymbol{r}$ is given by

$$
\frac{d \boldsymbol{v}}{d t}=\boldsymbol{g}
$$

with

$$
\boldsymbol{g}=-G \sum_{j}^{N} \frac{m_{j}}{\left\{\left(\boldsymbol{r}-\boldsymbol{r}_{j}\right)^{2}+\epsilon^{2}\right\}^{3 / 2}},
$$

where $G$ is the gravitational constant and $\epsilon$ is the softening parameter. We calculate the gravitational force $\boldsymbol{g}$ by using the "Remote-GRAPE" system (Nakasato, Mori \& Nomoto 1997) for which GRAPE refers to a special purpose computer for efficiently calculating the gravitational force and potential (Sugimoto et al. 1990). Self-gravity calculations can be performed in parallel with other calculations such as gasdynamics, star formation and feedback, so that the total calculation time is considerably shortened. The performance analysis is reported by Nakasato, Mori \& Nomoto (1997).

\subsection{Physical processes}

In each time step we calculate at each fluid point three time scales of the local gas dynamical time $\left(t_{\mathrm{dyn}}\right)$, the local cooling time $\left(t_{\mathrm{cool}}\right)$, and the sound crossing time $\left(t_{\mathrm{sound}}\right)$ :

$$
\begin{gathered}
t_{\mathrm{dyn}}=\sqrt{\frac{3 \pi}{32 G \rho_{\mathrm{g}}}}, \\
t_{\mathrm{cool}}=\frac{3}{2} \frac{1}{\mu^{2}(1-Y)^{2}} \frac{k_{\mathrm{B}} T}{n_{\mathrm{g}} \Lambda},
\end{gathered}
$$

and

$$
t_{\text {sound }}=\frac{l}{c_{\mathrm{s}}}
$$

where $\rho_{\mathrm{g}}=\mu m_{\mathrm{H}} n_{\mathrm{g}}, \mu$ is the mean molecular weight, $m_{\mathrm{H}}$ is the hydrogen mass, $n_{\mathrm{g}}$ is the gas number density, $T$ is the gas temperature, $Y(=0.25)$ is the helium mass fraction, $k_{\mathrm{B}}$ is the Boltzmann constant, $c_{\mathrm{s}}$ is local sound speed, and $l$ is the local scale length of the fluid. Here we set $l$ to be equal to the smoothing 
length of a gas particle. In our simulation stars are assumed to form in the rapidly cooling, Jeans-unstable, and converging region subject to

$$
t_{\mathrm{cool}}<t_{\mathrm{dyn}}<t_{\text {sound }} \text { and } \nabla \cdot \boldsymbol{v}<0 \text {. }
$$

Once a region simultaneously satisfying these criteria has been identified, we create new collisionless star particles there at a rate determined by local gas properties. The subsequent motion of star particles thus formed is determined only by gravity.

The criteria in equation (11) are similar to those used by Katz (1992), Navarro \& White (1993), and Steinmetz \& Müller (1994). However, they prescribed that about one third or half of the mass of a gas particle forms a new collisionless star particle and the rest is heated by supernovae. In contrast, we do not fix the mass fraction of a gas particle that is converted to a new star particle. We assume that the star formation rate (SFR) is proportional to the local gas density and inversely proportional to the local dynamical time:

$$
\frac{d \rho_{\mathrm{s}}}{d t}=C \frac{\rho_{\mathrm{g}}}{t_{\mathrm{dyn}}}
$$

where the SFR coefficient $C$ is treated as a free parameter according to the analysis by Katz, Weinberg \& Hernquist (1995). We note that our simulation is insensitive to the adopted value of this parameter (cf. $\S 3.1)$.

We estimate the mass of a newly born star particle as

$$
m_{\mathrm{s}}=\left\{1-\exp \left(-C \frac{\triangle t}{t_{\mathrm{dyn}}}\right)\right\} \pi l^{3} \rho_{\mathrm{g}}
$$

where $\Delta t$ is the time step used (Mori, Nakasato \& Nomoto 1998). Given the slope index $x$ and lower and upper mass limits $\left(m_{1}, m_{\mathrm{u}}\right)$ for the initial stellar mass function (IMF), the number of Type II supernovae (SNe II) progenitors for a star particle with mass $m_{\mathrm{s}}$ is calculated as

$$
N_{\mathrm{SN}}=\frac{x-1}{x} \frac{1-\left(m_{\mathrm{SN}, \mathrm{l}} / m_{\mathrm{u}}\right)^{-x}}{1-\left(m_{\mathrm{l}} / m_{\mathrm{u}}\right)^{1-x}} \frac{m_{\mathrm{s}}}{m_{\mathrm{u}}}
$$

where $m_{\mathrm{SN}, 1}=8 M_{\odot}$ is the lower mass limit of stars that will explode as SNe II. These IMF parameters affect the heating rate of interstellar medium and the ejection rate of heavy elements from the star particle. The number of SNe II is the most sensitive to the IMF slope among others. Current resolution in our simulation gives $m_{\mathrm{s}} \sim 10^{4-6} M_{\odot}$ far exceeding a typical mass of single stars. Therefore, we distribute the associated mass of the star particle over approximately $10^{2-4}$ single stars according to Salpeter's (1955) IMF $(x=1.35)$ provided that the lower and upper mass limits are taken as $m_{1}=0.1 M_{\odot}$ and $m_{\mathrm{u}}=60 M_{\odot}$, respectively.

Stars once formed work as sources of transferring the energy, synthesized heavy elements, and materials $(\mathrm{H}$ and $\mathrm{He})$ to the interstellar medium through supernovae or stellar winds from massive stars. This feedback process is most critical in the simulation of galaxy formation. However, previous authors adopted different treatments, mostly because there is no good appreciation of how it should be modeled in the SPH algorithm (Katz 1992; Navarro \& White 1993; Mihos \& Hernquist 1994).

In this paper, when a star particle is formed and identified with a stellar assemblage as described above, stars more massive than $8 \mathrm{M}_{\odot}$ start to explode as SNe II with the explosion energy of $10^{51}$ ergs 
and their outer layers are blown out with synthesized heavy elements into the interstellar medium leaving the $1.4 \mathrm{M}_{\odot}$ remnant. We can regard this stellar assemblage as continuous energy release at an average rate of $L_{\mathrm{SN}}=8.44 \times 10^{35} \mathrm{ergs} \mathrm{s}^{-1}$ per star during the explosion period from $\tau\left(m_{\mathrm{u}}\right)=5.4 \times 10^{6} \mathrm{yr}$ until $\tau\left(8 M_{\odot}\right)=4.3 \times 10^{7} \mathrm{yr}$, where $\tau(m)$ is the lifetime of a star of mass $m$ (David, Forman \& Jones 1990). Prior to the onset of SNe II explosions, however, their progenitors develop stellar winds and also release the energy of $10^{50}$ ergs into the interstellar medium at an average rate of $L_{\mathrm{SW}}=7.75 \times 10^{34} \mathrm{ergs} \mathrm{s}^{-1}$ per star. The released energy from stellar winds is supplied to the gas particles within a sphere of radius $R_{\mathrm{snr}}$, and the energy, heavy elements and materials from SNe II are subsequently supplied to the same region. The radius $R_{\mathrm{snr}}$ is set to be equal to the maximum extension of the shock front in the adiabatic phase of supernova remnant and given by Shull \& Silk (1979),

$$
R_{\mathrm{snr}}=32.9\left(\frac{E}{10^{51} \mathrm{ergs}}\right)^{1 / 4}\left(\frac{n_{\mathrm{g}}}{1 \mathrm{~cm}^{-3}}\right)^{-1 / 2} \mathrm{pc},
$$

where $E$ is the released energy. In each time step we estimate $n_{\mathrm{g}}$ equal to the number density of the surrounding gas of the star particle with the minimum limit of $10^{-4} \mathrm{~cm}^{-3}$. The gas within $R_{\mathrm{snr}}$ remains adiabatic until the multiple SNe II phase ends at $\tau\left(8 M_{\odot}\right)$, and then it cools according to the adopted cooling rate of the gas.

Tsujimoto et al. (1996) tabulated the masses of 27 heavy elements synthesized in the progenitors of SN Ia and II, and determined the number ratio of Type Ia supernovae (SNe Ia) relative to SNe II that best reproduces the observed abundance pattern among heavy elements in the solar neighborhood as well as in the Large and Small Magellanic Clouds. Given the IMF, the total number of SNe Ia and II from a star particle is easily estimated, and this number can then be related to the mass of ejected heavy elements from a star particle by making use of Table 2 of Tsujimoto et al. (1996). We note that our analysis in this paper is restricted to the metal enrichment by SNe II only, and the full analysis including the contribution from SNe Ia will be given elsewhere.

We compute the evolution of spectral energy distribution (SED) of a star particle based on the method of stellar population synthesis which utilizes the stellar evolutionary tracks for various masses and metallicities of stars (Arimoto \& Yoshii 1986). Using the updated version by Kodama \& Arimoto (1997), we calculate the SED for $\lambda=300 \AA-40000 \AA$ as a function of elapsed time from the formation of each star particle. The SED of a whole galaxy is then obtained by summing up SED's of all star particles ever formed at different times with different metallicities. In this paper the response functions for the $U B V R I$ passbands are taken from Bessell (1990) and those for the JHKL magnitudes from Bessell \& Brett (1988).

\subsection{Virialized protogalaxy}

Following a standard scenario of the CDM universe $\left(\Omega_{0}=1, H_{0}=50 \mathrm{~km} \mathrm{~s}^{-1} \mathrm{Mpc}^{-1}\right)$, we consider a protogalaxy as originated from the CDM density fluctuation with $\delta M / M$ equal to $\nu$ times the $r m s$ value $\sigma$. The fluctuation is normalized to unity for a spherical top-hat window of comoving radius $16 \mathrm{Mpc}$ with a bias parameter of $b=1$. We assume that this protogalaxy is composed of $10 \%$ baryon and $90 \%$ dark matter in mass and is initially in virial equilibrium.

A protogalaxy with the total mass $M$ is assumed to have the density profile of a singular isothermal

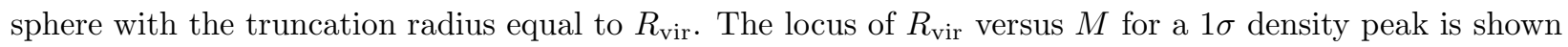
by thin line in Fig. 2 (cf. Bardeen et al. 1986). The local dynamical time of the singular isothermal sphere 
at the radius of $r$ is given by

$$
\tau_{\text {dyn }}(r)=\left(\frac{3 \pi^{2} R_{\mathrm{vir}}}{8 G M}\right)^{\frac{1}{2}} r
$$

and the local gas cooling time is given by

$$
\tau_{\text {cool }}(r)=\frac{2 \pi G m_{\mathrm{H}}^{2}}{(1-Y)^{2} F} \frac{1}{\Lambda} r^{2}
$$

where $F(=0.1)$ is the baryonic fraction. Equating $\tau_{\mathrm{dyn}}(r)$ to $\tau_{\text {cool }}(r)$, we define the cooling radius as

$$
R_{\mathrm{cool}}(M)=\left(\frac{3 R_{\mathrm{vir}}}{32}\right)^{\frac{1}{2}} \frac{(1-Y)^{2} F \Lambda}{m_{\mathrm{H}}^{2} G^{\frac{3}{2}}} M^{-\frac{1}{2}} .
$$

The $R_{\text {cool }}$ versus $M$ relation for the solar-abundance gas is shown by the upper thick line and the relation for the primordial gas is shown by the lower thick line in Fig. 2. In a region above (below) the locus of $R_{\text {cool }}(M)$, the cooling time is longer (shorter) than the dynamical time. For a less massive protogalaxy with $M<7 \times 10^{10} M_{\odot}$, the cooling is efficient over a whole range of $r$, so that these galaxies can cool and condense leading to the burst of star formation. On the other hand, for $M>7 \times 10^{10} M_{\odot}$, the star formation occurs only in the cooling region inside the radius of $R_{\text {cool }}\left(<R_{\mathrm{vir}}\right)$. In a particular case of $M=2 \times 10^{12} M_{\odot}$, the cooling region inside $R_{\text {cool }}$ contains about $5 \%$ of the total mass.

It is clear from Fig. 2 that the cooling is more efficient for higher metallicity. Since synthesized heavy elements by SNe II spread out due to the feedback process and stellar motions, the cooling region in which star formation occurs expands towards an outer part of the system. Accordingly this expansion of cooling region induces the formation of massive ellipticals even from the initial condition of low densities like $1 \sigma$-peaks.

\section{SIMULATIONS}

The formation of the CDM halos through the hierarchical clustering has been investigated with $N$-body simulations. Dubinski \& Carlberg (1991) argued that their equilibrium density profile is fitted by $\rho \propto r^{-1}(r+a)^{-3}$ or Hernquist's (1990) profile which has a central cusp and resembles de Vaucouleurs' $r^{1 / 4}$-profile in projection. Navarro, Frenk \& White (1997), however, pointed out that their structure can be approximated as $\rho(r) \propto r^{-1}(r+a)^{-2}$, which is more extended than the Hernquist profile. Fukushige \& Makino (1997) used a high-resolution simulation and showed that the CDM halos have a steeper central cusp than quoted above.

The resulting structure of the CDM halos depends on the number of particles used in the simulation.

Besides, there is no definitive view about whether the baryonic component has the same equilibrium profile as the dark matter halo. Thus, in this paper, we assumed that both baryon and dark matter initially have the King profile with the central concentration index of $c=2$ (Fig. 3; cf. Binney \& Tremaine 1987). Since our prime motivation is to clarify the effect of energy feedback in the evolution of galaxies, we simply neglect the possible mass-dependent profile of dark halos (Navarro, Frenk \& White 1997) and their angular momentum distribution (Mao \& Mo 1998). This two-component system is made to settle in a virial equilibrium from which the gas temperature and the velocity dispersion of dark matter are estimated. Our simulation uses $3 \times 10^{4}$ gas particles and the same number of dark matter particles to set up an initial condition. As the number of star particles increases due to star formation, the total number of particles increases up to about $10^{5}$ particles in the end of our simulation. 


\subsection{Dwarf elliptical galaxies}

We consider a less massive protogalaxy having a total mass of $10^{10} M_{\odot}$ with a baryon to dark matter ratio of $1 / 9$. The tidal radius of the King profile is $8.45 \mathrm{kpc}$. The mean density of the total system is

$2.7 \times 10^{-25} \mathrm{~g} \mathrm{~cm}^{-3}$, the mean temperature of the gas is $10^{5.1} \mathrm{~K}$, the mean velocity dispersion of dark matter is $72 \mathrm{~km} \mathrm{~s}^{-1}$, the mean dynamical time is $1.3 \times 10^{8} \mathrm{yrs}$, and the mean cooling time is $7.6 \times 10^{7} \mathrm{yrs}$. The gravitational softening parameter is adopted as $0.02 \mathrm{kpc}$ for gas particles, $0.05 \mathrm{kpc}$ for dark matter, and $0.03 \mathrm{kpc}$ for star particles.

Owing to the efficient radiative cooling mainly through collisional excitation of $\mathrm{H}$ and $\mathrm{He}^{+}$, the gas temperature rapidly drops, which induces a dynamical contraction of gas and dark matter by the self-gravity. When the gas temperature becomes close to $10^{4} \mathrm{~K}$ and stops decreasing, a quasi-isothermal contraction is established. The density in the central region increases by the accretion of the surrounding gas, and eventually the intensive star formation is triggered. When massive stars begin to explode as $\mathrm{SNe}$ II, the gas in the vicinity of SNe II acquires the thermal energy and synthesized heavy elements released from SNe II. Then, the gas temperature locally increases up to about $10^{7.5} \mathrm{~K}$, and subsequent formation of stars is virtually halted. About $5 \%$ of the initial gas mass is used up in this formation of the first generation stars.

Figure 4 shows the snapshots for the projected particle positions and the integrated energy spectra as a function of elapsed time from $0.5 \times 10^{7}$ to $1.0 \times 10^{9} \mathrm{yrs}$. The top three panels in each column show the spatial distributions of dark matter, gas, and stars, respectively, projected onto the $x-y$ plane. The bottom panel shows the spectral energy distribution (SED) from stellar populations in the evolving galaxy. The supernova-driven gas flow is generated outwards from the center of the protogalaxy. This outflow collides with the inflow of the gas accreting from outside, and the high-density super-shell is eventually formed. Figure 5 shows the radial profiles of the gas density (top panel), the temperature (middle panel), and the radial velocity (bottom panel) at the elapsed time of $1.0 \times 10^{7} \mathrm{yrs}$. We find from this figure that the shock waves propagate outwards with the shock front at $\sim 0.5 \mathrm{kpc}$ and the hot cavity is created inside the super-shell.

While the gas is continuously swept up by the super-shell, the gas density further increases due to the enhanced cooling rate in the already dense shell. Then the secondary star formation begins within the super-shell, and subsequent SNe further accelerate the outward expansion of the shell. This situation is clearly understood from the second panels of Fig. 4 in a time sequence from $1.0 \times 10^{7}$ to $3.0 \times 10^{7}$ yrs. Star formation in the expanding shell continue for $\sim 5.0 \times 10^{7}$ yrs until the gas density in the shell becomes too low to form new stars. About $20 \%$ of the initial gas mass is turned into stars in this stage. Finally, the outflowing gas escapes from the gravitational potential of the whole system and is ejected into the intergalactic space. The final stellar system possesses $\sim 25 \%$ of the initial gas mass.

The stars initially have the velocity vectors of the gas from which they were formed. Therefore, the first generation stars have zero systematic velocity, but the later generation stars have a large outward radial velocity component. The oscillation of swelling and contraction of the stellar system continues for several $10^{8} \mathrm{yrs}$, and the system becomes settled in a quasi-steady state until $\sim 1$ Gyrs. Consequently the system has a large velocity dispersion and a large core. The surface mass distribution is approximately exponential and is more extended than de Vaucouleurs' $r^{1 / 4}$-profile.

Stars are formed for the most part before the gas is fully polluted to the yield value of the synthesized heavy elements. The average metallicity of the stars in the system is as low as $[\mathrm{Fe} / \mathrm{H}] \sim-2.4$. This 
metallicity is consistent with a range covered by the observations, but is much lower than those of normal galaxies (Dekel \& Silk 1986; Yoshii \& Arimoto 1987). One outstanding feature discovered by our simulation is a positive metallicity gradient in this system which is in sharp contrast to the observed negative gradient for massive galaxies (Carollo, Danziger \& Buson 1993). The star-forming site moves outwards with the expanding shell and the gas in this shell is gradually enriched with synthesized heavy elements from $\mathrm{SNe}$ II. Stars of later generations are necessarily born at larger radii with larger metallicities, leading to the emergence of a positive metallicity gradient in the resulting stellar system. Figure 6 shows the projected distribution of stellar metallicity at different elapsed times of $1.0 \times 10^{7}, 2.0 \times 10^{7}, 3.0 \times 10^{7}$, and $1.0 \times 10^{9}$ yrs. The distribution does not change with time and the system should keep such a metallicity gradient during the age of the universe.

Figure 7 shows the projected surface brightness distribution at 15 Gyrs in the $B, V, R, I$ and $K$ bands. These profiles are plotted against a linear scale of the radius in kpc or a quatic root of the radius in kpc. The resulting system is characterized by an exponential profile rather than de Vaucouleurs' $r^{1 / 4}$-profile. Figure 8 shows the projected color profiles at 15 Gyrs for $V-K$ (circles), $B-R$ (squares), $B-V$ (diamonds), and $V-R$ (triangles). These profiles are scaled vertically in this figure to coincide with each other at the galaxy center. The result is consistent with the observed trend of redder colors at larger radii for dwarf galaxies (Vader et al. 1988; Kormendy \& Djorgovski 1989; Chaboyer 1994).

In our simulation of dwarf ellipticals, the total stellar mass is smaller when a flatter IMF is adopted. However, the stellar metallicity is not so sensitive to the IMF slope and remains about one tenth of than the solar in agreement with observations of nearby dwarf galaxies. This is because for the flatter IMF the number of supernovae is larger so that the heated gas and heavy elements therein are blown out of the system at a larger rate.

We also examined how our simulation depends on the SFR coefficient $C$ in equation 12 . For $C=0.1$ and $C=1.0$, we carried out the simulations with the same initial condition. Our simulation run gives the total stellar mass of $3.12 \times 10^{8} M_{\odot}$ for $C=0.1$ and $4.27 \times 10^{8} M_{\odot}$ for $C=1.0$, indicating that the result is insensitive to the adopted value of $C$ as noted by Katz (1992) and Katz, Weinberg \& Hernquist (1995).

\subsection{Normal elliptical galaxies}

In this section we study the formation of a more massive system for the purpose of comparison with a less massive system of dwarf elliptical galaxies. We consider a protogalaxy having a total mass of $10^{12} M_{\odot}$ with a baryon to dark matter ratio of $1 / 9$. The tidal radius of the King profile is $82.8 \mathrm{kpc}$. The mean

density of the total system is $2.8 \times 10^{-26} \mathrm{~g} \mathrm{~cm}^{-3}$, the mean temperature of the gas is $10^{6.1} \mathrm{~K}$, and the mean velocity dispersion of dark matter is $228 \mathrm{~km} \mathrm{~s}^{-1}$, the mean dynamical time is $4.0 \times 10^{8} \mathrm{yrs}$, and the mean cooling time is $5.7 \times 10^{10} \mathrm{yrs}$. The gravitational softening parameter is adopted as $0.2 \mathrm{kpc}$ for gas particles, $0.5 \mathrm{kpc}$ for dark matter, and $0.3 \mathrm{kpc}$ for star particles.

Figure 9 shows the snapshots for the projected particle positions and the integrated energy spectra as a function of elapsed time from $0.1 \times 10^{9}$ to $2.0 \times 10^{9} \mathrm{yrs}$. The top three panels in each column show the spatial distributions of dark matter, gas, and stars, respectively, projected onto the $x-y$ plane. The bottom panel shows the SED from the evolving galaxy.

Contrary to dwarf ellipticals, a massive protogalaxy is considered to evolve from relatively low gas density and high virial temperature, so that the gas does not cool rapidly. The protogalaxy initially in virial 
equilibrium shrinks quasi statically, and the gas density near the galaxy center gradually increases. When the gas density rises sufficiently, the temperature suddenly drops due to the thermal instability. Figure 10 shows that the gas temperature drops down to about $10^{4} \mathrm{~K}$ and the gas density further increases in the central region where active star formation starts to occur.

Newly born massive stars heat up the surrounding gas and at the same time the rate of their formation is suppressed in the heated gas. Therefore, higher or lower rate of star formation is stabilized and self-regulated when all star-forming activities are confined within the deep gravitational potential of a massive galaxy.

Figure 11 shows the projected surface brightness distribution at 15 Gyrs plotted against a linear scale of the radius in $\mathrm{kpc}$ or a quatic root of the radius in kpc. The surface brightness distribution at 15 Gyr is similar to an $r^{1 / 4}$-profile, and the integrated blue luminosity is $M_{B}=-21.5 \mathrm{mag}$.

Since massive stars enrich the gas with synthesized heavy elements from which new stars are subsequently born, the stellar metallicity increases in proportion to the heavy element abundances in the gas. In the central region of the galaxy, cycles of stellar birth and death continue until the metallicity approaches the yield value, whereas in the outer part of the galaxy the gas is used up in star formation before it is significantly polluted. Consequently as shown in Fig. 12, there appears a negative gradient of stellar metallicity across the system corresponding to bluer colors at larger radii as observed in normal galaxies (e.g. Carollo, Danziger \& Buson 1993).

We however note that the average stellar metallicity is $[\mathrm{Fe} / \mathrm{H}] \sim-0.2$, which is about three times lower than the observed metallicity of $[\mathrm{Fe} / \mathrm{H}] \sim+0.3$, either because Salpeter's IMF adopted in our simulation is too steep or because an initial density of the protogalaxy taken from a $1 \sigma$ density peak is too low to form massive elliptical galaxies. Our numerical experiments indicate that a flatter IMF with $x=1.05$ is able to reproduce the observed metallicity. In order to refine the model, we need more extensive simulations from different initial conditions by including the iron supply from SNe Ia in the gas. Comprehensive analysis along this line will be reported elsewhere.

\section{SUMMARY AND CONCLUSION}

We have developed a three-dimensional $N$-body/SPH simulation code combined with stellar population synthesis and applied it to the study of dynamical, chemical and spectro-photometric evolution of dwarf and normal elliptical galaxies embedded in a dark matter halo. A protogalaxy is assumed to be a virialized non-rotating sphere in a $1 \sigma \mathrm{CDM}$ perturbation enclosing the total mass of $10^{10} M_{\odot}$ and $10^{12} M_{\odot}$ with $10 \%$ baryonic mass.

For dwarf ellipticals, the gas temperature rapidly decreases by the efficient cooling and the protogalaxy starts to collapse due to the self-gravity of gas and dark matter. When the gas density near the galaxy center becomes large, star formation burst takes place (SFR $\sim 23 M_{\odot} \mathrm{yr}^{-1}$ ) and the collapse is halted by supernova-driven wind from the central star-forming region. About $75 \%$ of the initial gas mass is then lost from the system on a much shorter time scale compared to the mean crossing time of a star in the system. Thereby the system expands and recovers a new equilibrium state as a loosely-bound stellar system exhibiting an exponential structure.

On the other hand, normal ellipticals are considered to evolve from ten times lower gas density and ten times higher virial temperature. The gas is thermally stable having a relatively longer cooling time. In our 
simulation, most of the gas is used up in formation of stars and only a small fraction of gas is evaporated out of the system in the form of irregular gaseous blobs. Since the time scale of this gas removal is much longer than the crossing time of a star, the stellar system remains in quasi-equilibrium and evolves into an $r^{1 / 4}$-structure as observed.

The dynamical evolution of the system is determined not only by the amount of gas removal but also by its time scale. These decisive factors of gas removal depend critically on the cooling efficiency which is systematically higher for less massive protogalaxies in the CDM universe (see Fig. 2). In particular their efficient cooling causes a significant dynamical impact in making an exponential structure of dwarf galaxies. However, in a low-mass end below $10^{8} M_{\odot}$, protogalaxies have much more efficient cooling, so that a large amount of gas is locked up in formation of the first-generation stars until they start to explode as supernovae. Consequently supernovae no longer affect the subsequent evolution of the stellar system and very low-mass galaxies could survive as first demonstrated by Yoshii \& Arimoto (1987).

It is therefore intriguing to simulate spheroidal stellar systems along their mass sequence from giant elliptical galaxies to compact globular clusters in order to see whether the energy feedback works to suppress the formation of dwarf galaxies only and modifies the shape of the luminosity function as observed.

We are grateful to T. Shigeyama, M. Chiba and T. Tsujimoto for many fruitful discussions, and to T. Kodama for providing us the tables of population synthesis. This work has been supported in part by the Research Fellowship of the Japan Society for the Promotion of Science for Young Scientists (6867), the Grant-in-Aid for Scientific Research (05242102, 06233101), and the Center-of-Excellence Research (07CE2002) of the Ministry of Education, Science, Sports, and Culture in Japan. The computations were mainly carried out on the "Remote-GRAPE" system at University of Tokyo and partly done on Fujitsu VPP-300 at the National Astronomical Observatory in Japan, and Fujitsu VPP-500 at the Institute of Physical and Chemical Research (RIKEN).

\section{REFERENCES}

Arimoto, N, \& Yoshii, Y. 1986, 164, 260

Babul, A. \& Ferguson, H. C. 1996, ApJ, 458, 100

Bardeen, J. M., Bond, J. R., Kaiser, N., \& Szalay, A. S. 1986, ApJ, 304, 15

Bessell, M. S. 1990, PASP, 102, 1181

Bessell, M. S., \& Brett, J. M. 1988, PASP, 100, 1134

Binggeli, B., \& Cameron, L. M. 1991, A\&A, 252, 27

Binggeli, B., \& Jerjen, H. 1998, A\&A, 333, 17

Binney, J., \& Tremaine, S. 1987, Galactic Dynamics. Princeton University Press, Princeton, p. 232

Caldwell, N., \& Bothun, G. D. 1987, AJ, 94, 1126

Carollo, C. M., Danziger, I. J., \& Buson, L. 1993, MNRAS, 265, 553

Chaboyer, B. 1994, in ESO/OHP Workshop on Dwarf Galaxies., eds. Meylan, G. \& Prugniel, P., ESO, Garching, 485

Chiba, M., \& Nath, B. B. 1994, ApJ, 436, 618 
Cole, S., Aragón-Salamanca, A., Frenk, C. S., Navarro, J. F. \& Zepf, S. E., 1994, MNRAS, 271, 781

David, L. P., Forman, W., \& Jones, C. 1990, ApJ, 359, 29

Davis, M., Efstathiou, G., Frenk, C., \& White, S. D. M. 1985, ApJ, 292, 371

Dekel, A., \& Silk, J. 1986, ApJ, 303, 39

de Vaucouleurs, G. 1948, Ann. d'Astrophys. 11, 247

Dubinski, J., \& Carlberg, R. 1991, ApJ, 378, 496

Durrell, P. R., McLaughlin, D. E., Harris, W. E., \& Hanes, D. A. 1996, ApJ, 463, 543

Efstathiou, G. 1992, MNRAS, 256,43P

Faber, S. M., \& Lin, D. M. C. 1983, ApJ, 266,L17

Fukushige, T., \& Makino, J. 1997, ApJ, 477, L9

Gingold, R. A., \& Monaghan, J. J. 1977, MNRAS, 181, 375

Hernquist, L. 1990, ApJ, 356, 359

Hunter, D. A. 1996, ApJ, 457, 671

Ichikawa, S., Wakamatsu, K., \& Okamura, S. 1986, ApJS, 60, 475

Katz, N. 1992, ApJ, 391, 502

Katz, N., Weinberg, D. H., \& Hernquist, L. 1996, ApJ, 463, 305

Kobayashi, C., Tsujimoto, T., Nomoto, K., Hachisu, I., \& Kato, M. ApJ, in press

Kodama, T., \& Arimoto, N. 1997, A\&A, 320, 41

Kormendy J. 1977, ApJ, 218, 333

Kormendy, J., \& Djorgovski, S. 1989, ARA\&A, 27, 235

Lacey, C., \& Silk, J. 1991, ApJ, 381, 14

Loveday, J. 1997, ApJ, 489, 29

Lucy, L. 1977, AJ, 82, 1013

Marlowe, A. T., Heckman, T. M., \& Wyse, R. F. G. 1995, ApJ, 438, 563

Meurer, G. R., Freeman, K. C., \& Dopita, M. A. 1992, AJ, 103, 60

Mihos, J. C., \& Hernquist, L. 1994, ApJ, 437, 611

Monaghan, J. J., \& Lattanzio, J. C. 1985, A\&A, 149, 135

Mao, S., \& Mo, H. J. 1998, MNRAS, 296, 847

Mori, M., Nakasato, N., \& Nomoto, K. 1998, in preparation.

Mori, M., Yoshii, Y., Tsujimoto, T., \& Nomoto, K. 1997, ApJ, 478, L21

Nakasato, N., Mori, M., \& Nomoto, K. 1997, ApJ, 484, 608

Navarro, J. F., Eke, V. R., \& Frenk, C. S. 1996, MNRAS, 283, L72

Navarro, J. F., Frenk, C. S., \& White, S. D. M. 1997, ApJ, 490, 493

Navarro J. F., \& White, S. D. M. 1993, MNRAS, 265, 271

Patterson, R. J., \& Thuan, T. X. 1996, ApJS, 107, 103 
Puche, D. \& Westpfahl, D. 1994, in ESO/OHP Workshop on Dwarf Galaxies., eds. Meylan, G. \& Prugniel, P., ESO, Garching, 273

Saito, M. 1979, PASJ, 31, 193

Salpeter, E. E. 1955, ApJ, 121, 161

Sandage, A., Binggeli, B., \& Tammann, G. A. 1985, AJ, 90, 1759

Shull, J. M., \& Silk, J. 1979, ApJ, 234, 427

Steinmetz, M., \& Müller, E. 1994, ApJ, 281, L97

Sugimoto, D., Chikada Y., Makino J., Ito T., Ebisuzaki T., \& Umemura M. 1990, Nature, 345,33

Sutherland, R. S., \& Dopita, M. A. 1993, ApJS, 88, 253

Theis, C. H., Burkert, A., \& Hensler, G. 1992, A\&A, 265, 465

Thoul, A. A., \& Weinberg, D. H. 1995, ApJ, 442, 480

Trentham, N. 1998, MNRAS, 294, 193

Tsujimoto, T., Nomoto, K., Yoshii, Y., Hoshimoto, M., Yanagida, S., \& Thielemann F. -K. 1996, MNRAS, 277,945

Vader, J. P., Vigroux, L., Lachièze-Rey, M., \& Souviron J. 1988, A\&A, 203, 217

van Albada, T. S. 1982, MNRAS, 201, 939

Vigroux, L., Souviron J., Lachièze-Rey, M., \& Vader, J. P. 1988, A\&AS, 73, 1

Westpfahl, D., \& Puche, D. 1994, in ESO/OHP Workshop on Dwarf Galaxies., eds. Meylan, G. \& Prugniel, P., ESO, Garching, 295

White, S. D. M. 1979, MNRAS, 189, 831

White, S. D. M., \& Frenk, C. S. 1991, ApJ, 379, 52

Yoshii, Y., \& Arimoto, N. 1987, A\&A, 188, 13

Young, C. K., \& Currie, M. J. 1994, MNRAS, 268, L11

This preprint was prepared with the AAS $\mathrm{LAT}_{\mathrm{E}} \mathrm{X}$ macros v4.0. 
Fig. 1.- The cooling rate $\Lambda$ divided by the square of number density $n$ of gas particles is plotted against the temperature. Each curve shows the cooling rate for the value of $[\mathrm{Fe} / \mathrm{H}]$ indicated. The bottom curve corresponds to the zero metallicity.

Fig. 2.- Radius of a protogalaxy as a sequence of the total mass with a baryon to dark matter ratio equal to $1 / 9$. Thick lines show the cooling radius $R_{\text {cool }}$ from $\tau_{\text {dyn }}=\tau_{\text {cool }}$ for the solar metallicity (upper line) and the zero metallicity (lower line). Thin line shows the virial radius $R_{\text {vir }}$ from a $1 \sigma$ density perturbation in the standard CDM universe $\left(\Omega_{0}=1, H_{0}=50 \mathrm{~km} \mathrm{~s}^{-1} \mathrm{Mpc}^{-1}\right)$.

Fig. 3.- Initial density profile of dark matter having the mass of $9 \times 10^{9} M_{\odot}$. The solid line indicates the exact density distribution of the King profile with the central concentration index of $c=2$, and the crosses are the initial densities set up in our simulation. The inset shows the projected particle distribution in the $x-y$ plane.

Fig. 4.- Snapshots for the projected particle positions and the spectral energy distribution (SED) at five different elapsed times for our simulation run of a protogalaxy with the total mass of $10^{10} M_{\odot}$. The top three panels in each column show the spatial distributions of dark matter, gas, and stars, respectively, projected onto the $x-y$ plane. The bottom panel shows the SED from stellar populations in the evolving galaxy.

Fig. 5. - Snapshot for the protogalaxy with the total mass of $10^{10} M_{\odot}$ at the elapsed time of $1.0 \times 10^{7}$ yrs. Shown are the radial profile of the gas density (top panel), the gas temperature (middle panel), and the radial component of the gas velocity (bottom panel).

Fig. 6.- Projected distributions of stellar metallicity $[\mathrm{Fe} / \mathrm{H}]$ at four different elapsed times for our simulation run of a protogalaxy with the total mass of $10^{10} M_{\odot}$.

Fig. 7.- Projected distribution of surface brightness at 15 Gyrs for our simulation run of a protogalaxy with the total mass of $10^{10} M_{\odot}$. Thick lines from top to bottom are the surface brightnesses in the $B, V, R$, $I$ and $K$ bands, respectively, plotted against a liner scale of the radius in kpc or a quatic root of the radius in kpc. A mean $B$-band distribution observed for a majority of dwarf galaxies is also shown for reference (e.g. Patterson \& Thuan 1996).

Fig. 8.- Projected distribution of integrated color at 15 Gyrs for our simulation run of a protogalaxy with the total mass of $10^{10} M_{\odot}$. Shown are the colors of $V-K$ (circles), $B-R$ (squares), $B-V$ (diamonds), and $V-R$ (triangles). Their values are offset at the galaxy center. The inset shows the projected distribution of stellar metallicity at 15 Gyrs

Fig. 9.- Snapshots for the projected particle positions and the spectral energy distribution (SED) at five different elapsed times for our simulation run of a protogalaxy with the total mass of $10^{12} M_{\odot}$. The top three panels in each column show the spatial distributions of dark matter, gas, and stars, respectively, projected onto the $x-y$ plane. The bottom panel shows the SED from stellar populations in the evolving galaxy.

Fig. 10.- Snapshot for the protogalaxy with the total mass of $10^{12} M_{\odot}$ at the elapsed time of $0.5 \times 10^{9}$ yrs. Shown are the radial profile of the gas density (top panel), the gas temperature (middle panel), and the radial component of the gas velocity (bottom panel).

Fig. 11.- Projected distribution of surface brightness at 15 Gyrs for our simulation run of a protogalaxy with the total mass of $10^{12} M_{\odot}$. Thick lines from top to bottom are the surface brightnesses in the $B, V, R$, $I$ and $K$ bands, respectively, plotted against a liner scale of the radius in kpc or a quatic root of the radius 
in kpc.

Fig. 12.- Projected distribution of integrated color at 15 Gyrs for our simulation run of a protogalaxy with the total mass of $10^{12} M_{\odot}$. Shown are the colors of $V-K$ (circles), $B-R$ (squares), $B-V$ (diamonds), and $V-R$ (triangles). Their values are offset at the galaxy center. The inset shows the projected distribution of stellar metallicity at 15 Gyrs. 


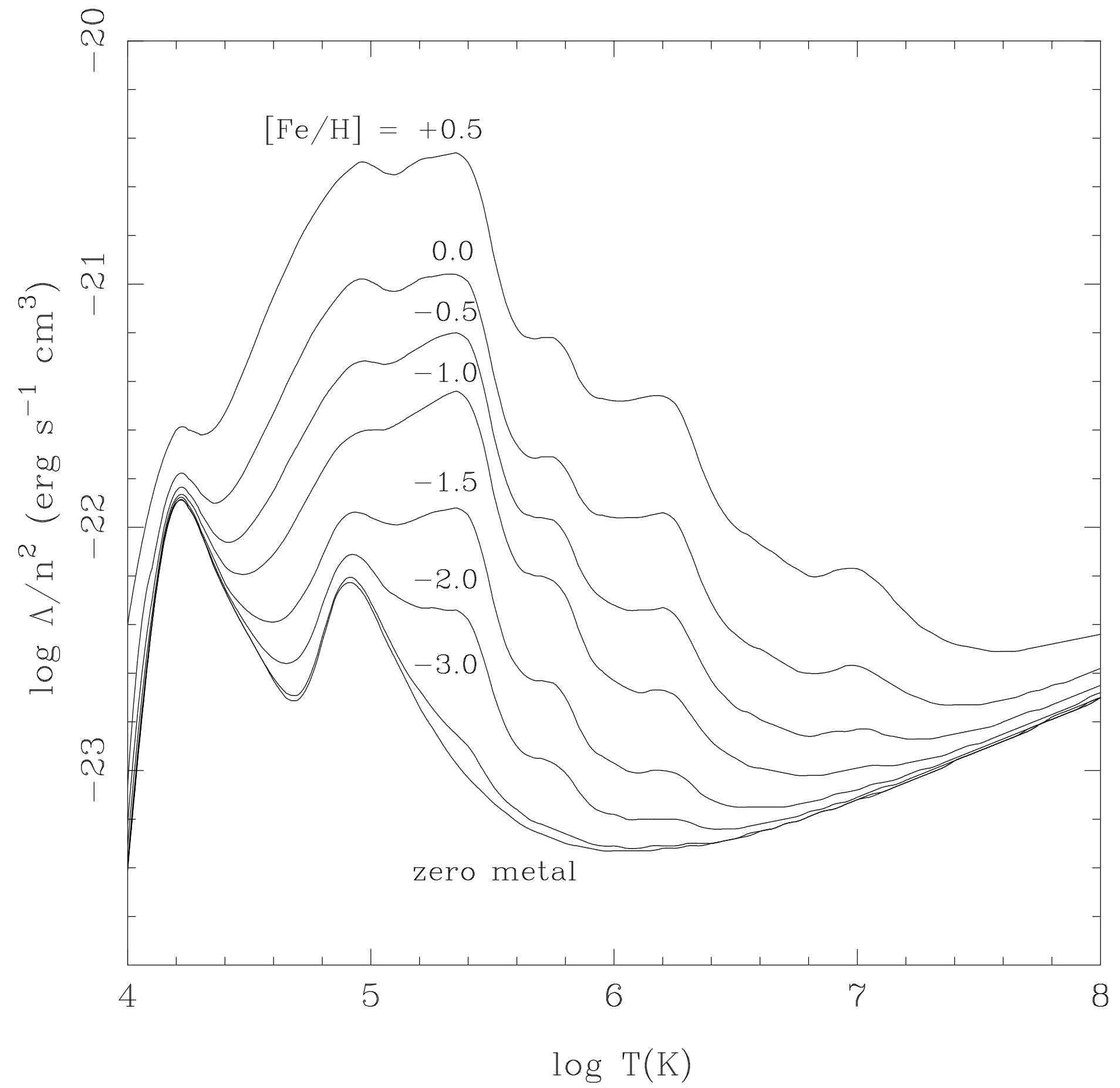




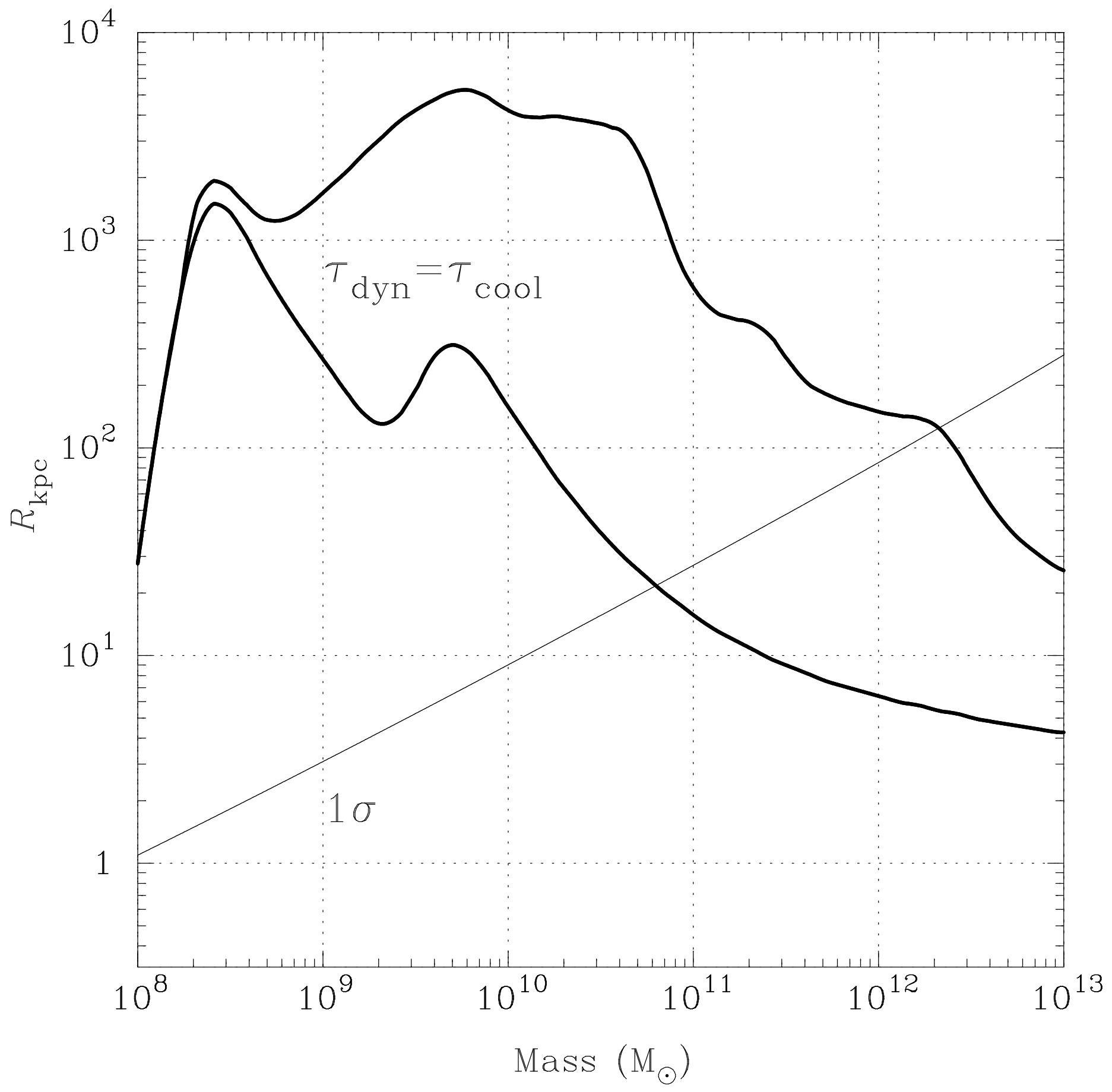


This figure "f03.jpg" is available in "jpg" format from: http://arxiv.org/ps/astro-ph/9812282v1 
This figure "f04.jpg" is available in "jpg" format from: http://arxiv.org/ps/astro-ph/9812282v1 
This figure "f05.jpg" is available in "jpg" format from: http://arxiv.org/ps/astro-ph/9812282v1 


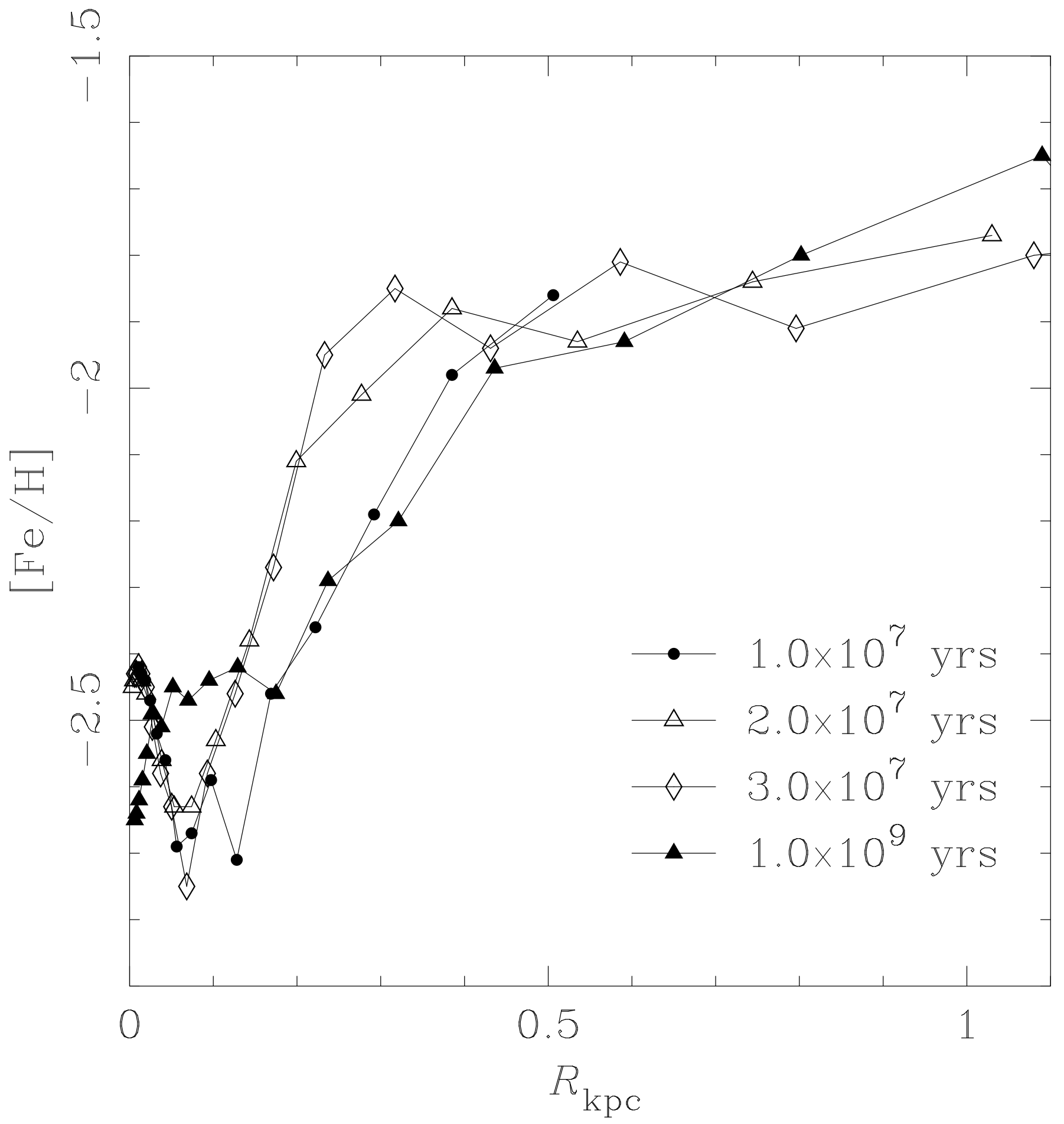




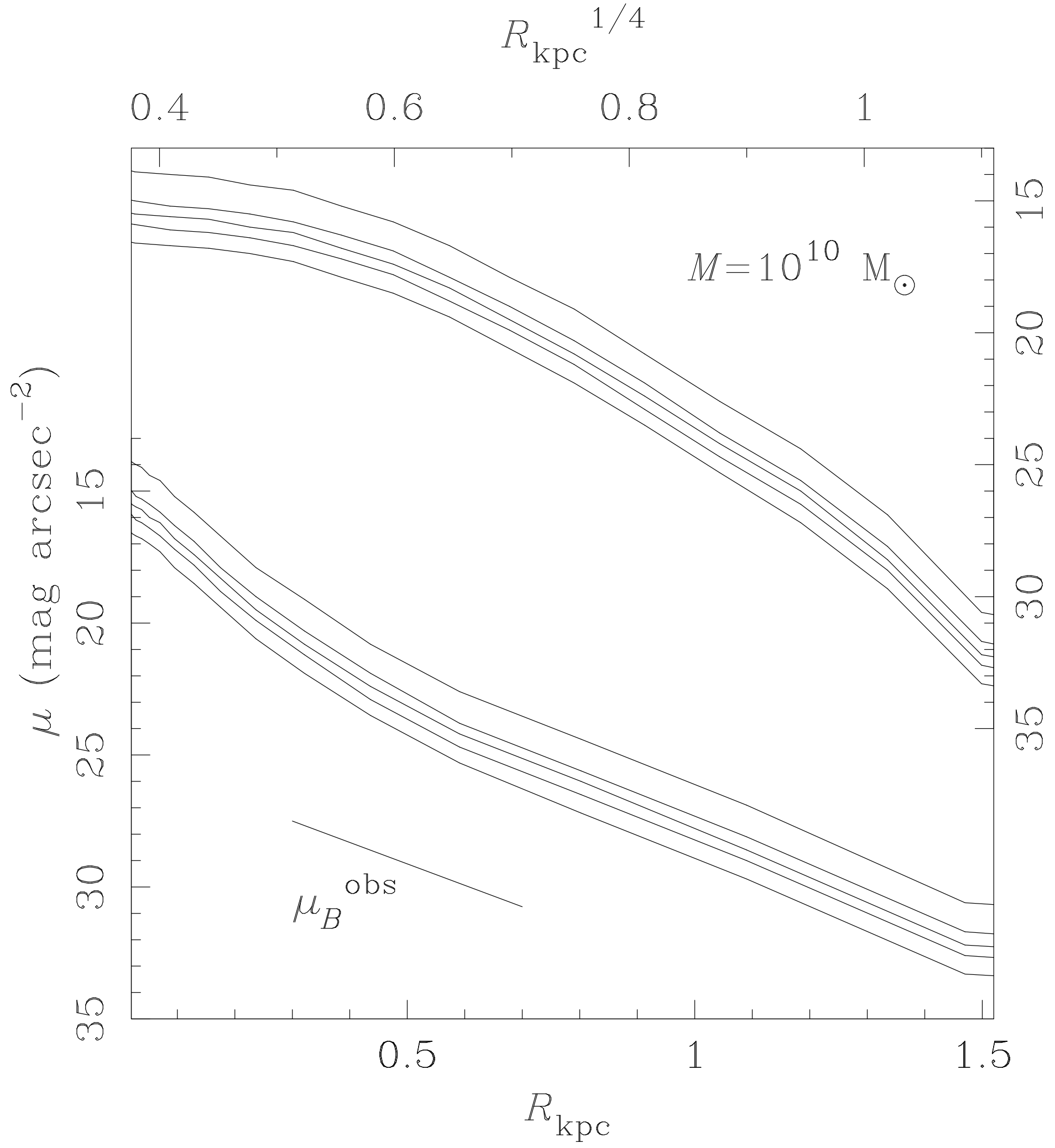




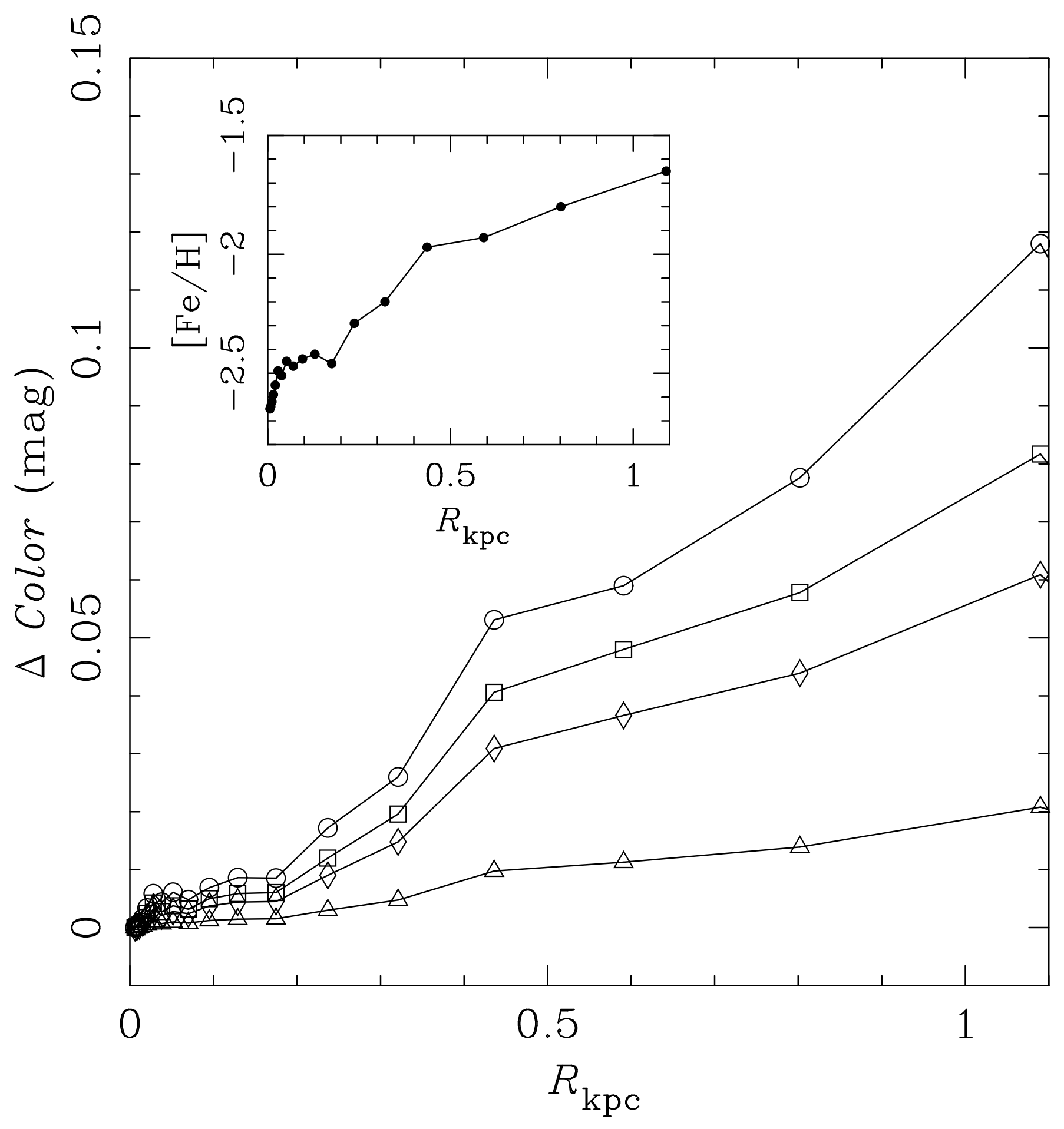


This figure "f09.jpg" is available in "jpg" format from: http://arxiv.org/ps/astro-ph/9812282v1 


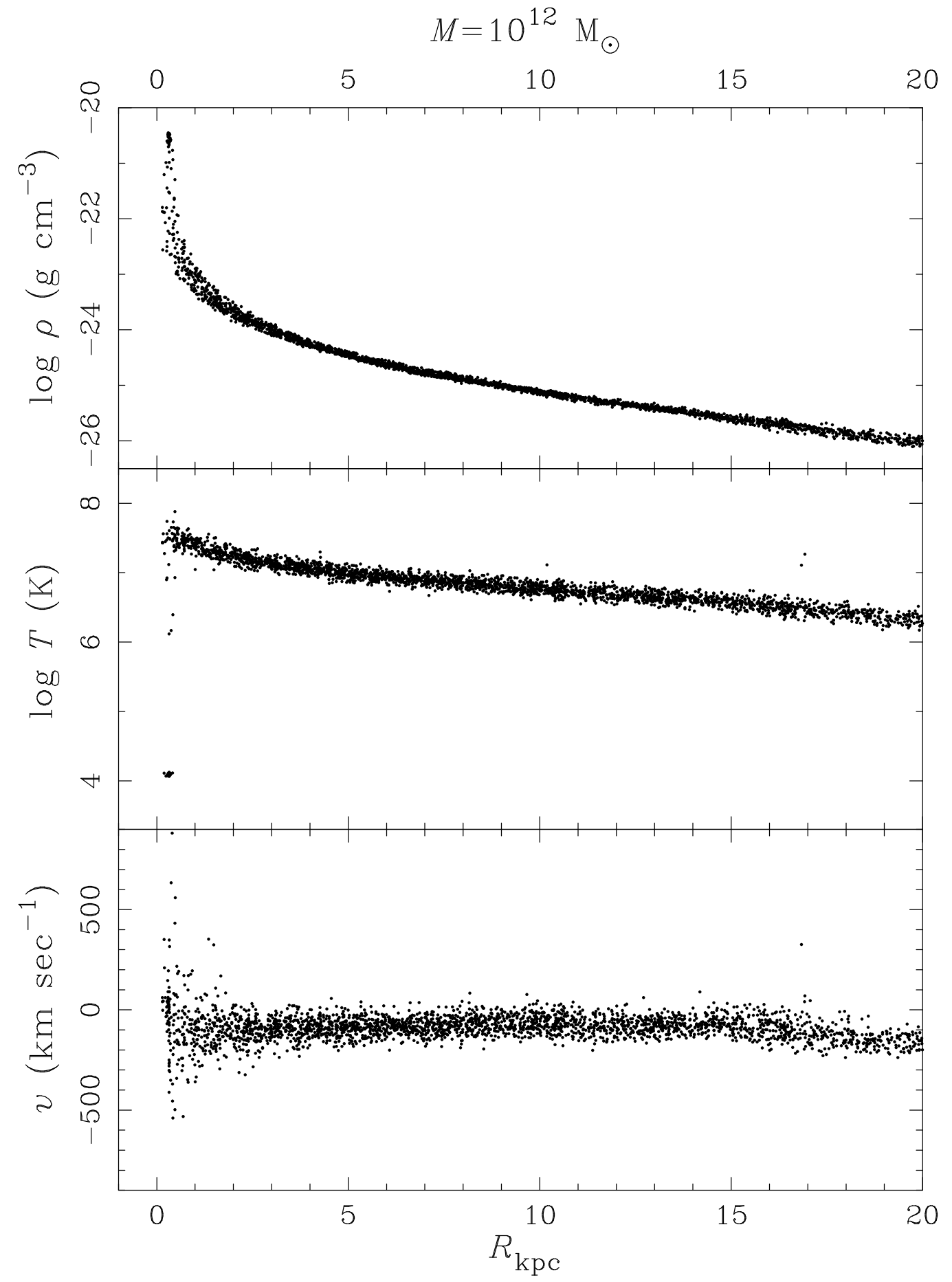




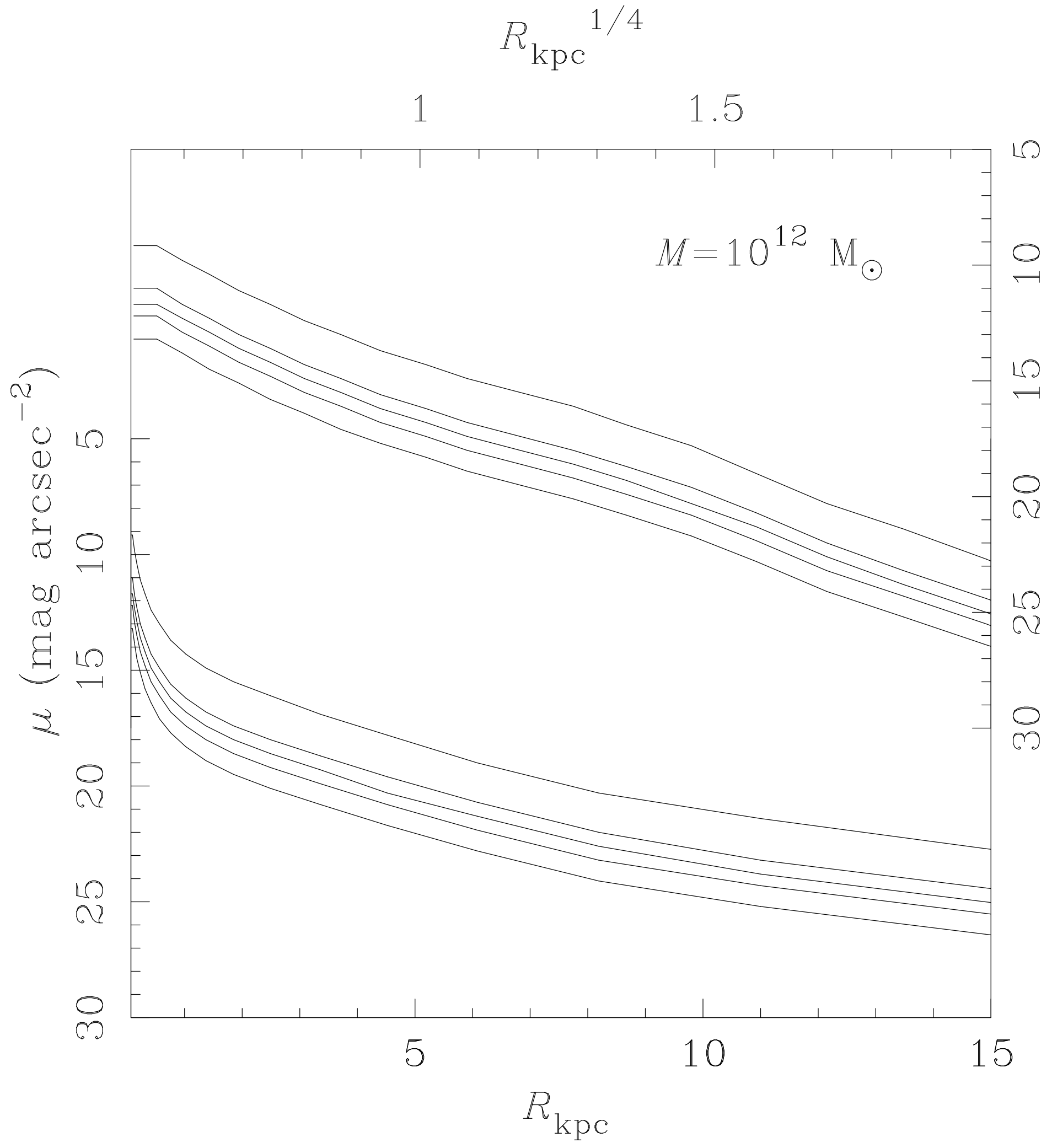




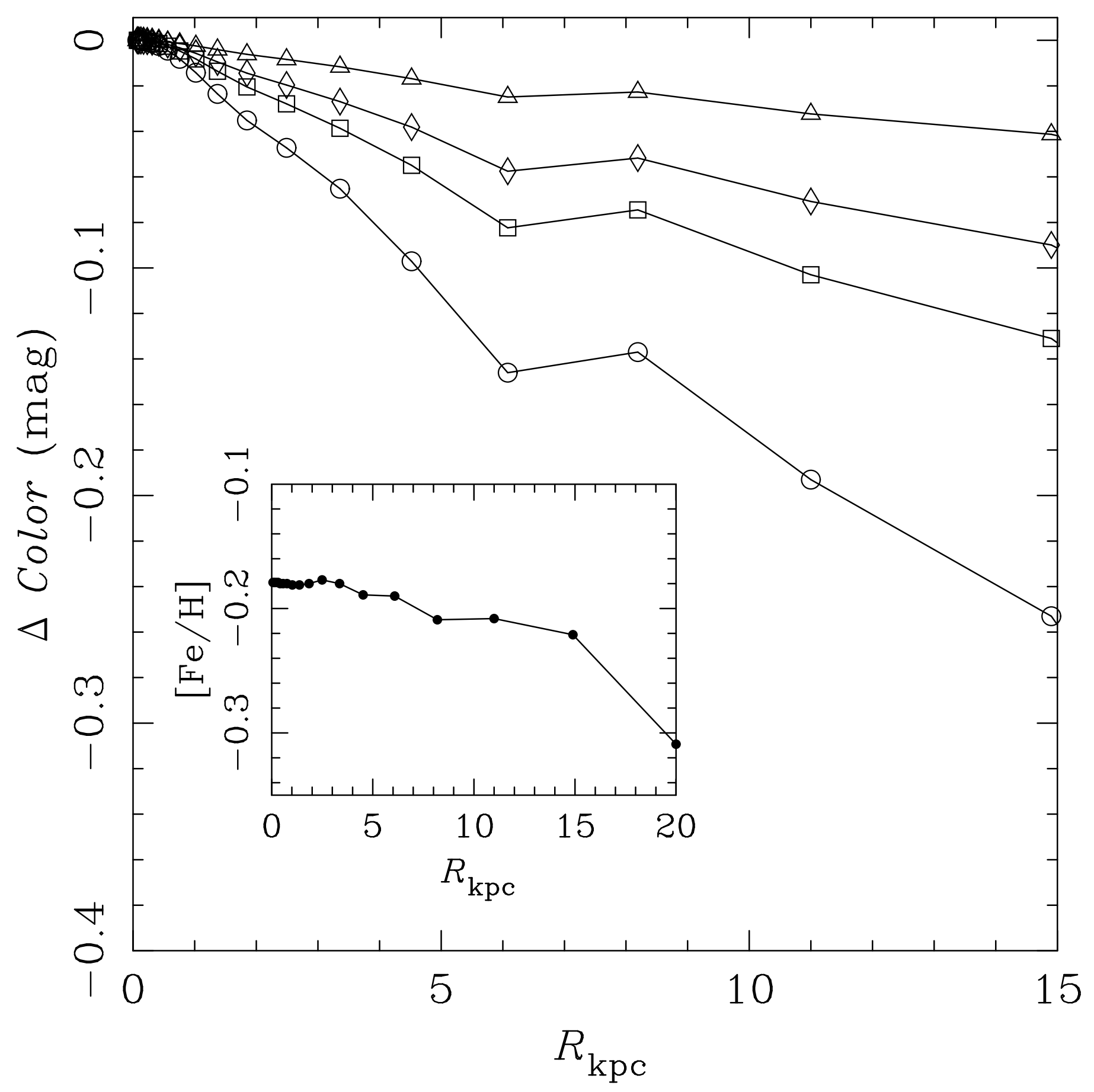

